\title{
Wie hängen Lohnhöhe und Beschäftigung zusammen?
}

Der Zusammenhang zwischen Lohn- und Beschäftigungsentwicklung ist eine theoretisch umstrittene Frage. Die verschiedenen Denkschulen stimmen nicht nur bezüglich der Größenordnungen der Effekte nicht überein, sondern ziehen zum Teil auch unterschiedliche Wirkungskanäle heran und betrachten unterschiedliche Zeiträume. Daher wird sogenannten evidenzbasierten Studien besondere Aufmerksamkeit geschenkt, um die Frage zu beantworten, wie sich eine Lohnerhöhung letztendlich auf die Beschäftigung auswirkt. Das makroökonometrische Mehr-Länder-Modell NiGEM wurde in den letzten Jahrzehnten in der wirtschaftspolitischen Beratung in diesem Kontext vielfach genutzt, auch von der Deutschen Bundesbank und dem Sachverständigenrat zur Begutachtung der gesamtwirtschaftlichen Entwicklung (SVR). Dieser Beitrag knüpft an die Bedeutung des NiGEM-Modells in der wirtschaftspolitischen Beratung an und prüft, inwieweit der dort modellierte Zusammenhang zwischen Lohnhöhe und Beschäftigung empirisch valide ist.

Die Analysen dieses Beitrags basieren auf dem vom National Institute of Economic and Social Research (NIESR) entwickelten Modell NiGEM (Hantzsche, Lopresto und Young, 2018). Es ist ein umfassendes Simulations- und Prognosemodell für die Weltwirtschaft, das typische neukeynesianische Elemente wie Preis- und Lohnrigiditäten und eine um Inflationserwartungen erweiterte PhillipsKurve umfasst. Die Erwartungsbildung der Wirtschaftsakteure erfolgt für Prognosen adaptiv; Simulationen können alternativ auch unter rationalen Erwartungen ausgeführt werden. Das Modell ermöglicht eine konsistente und dennoch detaillierte Abbildung der volkswirtschaftlichen Gesamtrechnungen (VGR) aller OECD- und zahlreicher Schwellenländer. Die übrigen Wirtschaftsräume der Welt werden in regionalen Blöcken modelliert, sodass das Wachstum der Importvolumina dem Wachstum der Exportvolumina auf globaler Ebene entspricht.

\section{Arbeitsmarktmodellierung in NiGEM}

NiGEM versteht sich als ein makroökonometrisches Modell zwischen zwei extremen Modellierungsansätzen ( $\mathrm{Pa}$ gan, 2003): Im Gegensatz zu Simulationsmodellen, etwa vielen DSGE (dynamic stochastic general equilibrium)Modellen, die eine rein kalibrierte Modellklasse bilden,

(C) Der/die Autor(en) 2020. Open Access: Dieser Artikel wird unter der Creative Commons Namensnennung 4.0 International Lizenz (https:// creativecommons.org/licenses/by/4.0/deed.de) veröffentlicht.

Open Access wird durch die ZBW - Leibniz-Informationszentrum Wirtschaft gefördert. werden die Parameter im NiGEM mit Hilfe eines Datensatzes geschätzt. Eine vollkommen freie Schätzung im Sinne von Vektorautoregressiven (VAR) Modellen ist angesichts der Zahl der Variablen aber nicht realisierbar und de facto finden Ausschlussrestriktionen durch die Variablenauswahl in den Gleichungen statt. In vorliegendem Beitrag werden die drei Gleichungen des Lohn-Preis-Beschäftigungssystems näher analysiert, da die Auswirkungen von Lohnsteigerungen auf die Beschäftigung hauptsächlich dort verankert sind. Im NiGEM-Modell legt die Profitmaximierung der Firmen grundsätzlich Arbeits- und Kapitalnachfrage fest, sodass das Arbeits-Outputverhältnis negativ vom Reallohn und technischen Fortschritt und das

Dr. Thomas Theobald ist Referatsleiter für Finanzmärkte und Konjunktur im Institut für Makroökonomie und Konjunkturforschung (IMK) der Hans-BöcklerStiftung.

Dr. Rudolf Zwiener, promovierter Makroökonom, war dort Referatsleiter.

Prof. Dr. Camille Logeay lehrt Volkswirtschaftslehre an der Hochschule für Technik und Wirtschaft in Berlin. 
Kapital-Outputverhältnis negativ von den realen Kapitalnutzungskosten abhängen. Da die hierdurch implizierte negative Beziehung zwischen Lohnhöhe und Beschäftigung Eingang in alle aggregierten Arbeitsmarktgleichungen findet, folgt NiGEM grundsätzlich dem neoklassischen Ansatz. Alle drei Gleichungen des Arbeitsmarktsystems nehmen die Form einer Fehlerkorrektur an und teilen eine ähnliche Langfristbeziehung (blau hervorgehoben in Kasten 1) zwischen Beschäftigung, Output, technologischem Fortschritt sowie Löhnen und Preisen. In der Lohngleichung spielt zudem die Arbeitslosigkeit eine Rolle. In der Preisgleichung wird ein kapazitätsauslastungsabhängiger Markup über den marginalen Kosten von Löhnen und Kapital herangezogen.

Die drei Gleichungen im Kasten 1 implizieren durch die Auswahl der Variablen in der langen wie in der kurzen Frist Ausschlussrestriktionen (Sims, 1980). Die Koeffizienten in den Langfristbeziehungen unterliegen mehreren Restriktionen. Beispielsweise ergibt sich eine langfristige Elastizität zwischen Reallohn und Arbeitsproduktivität von 0,5. In der Lohngleichung des Systems entspricht das $\mathrm{c} 14=$ $-\mathrm{c} 16$ und $\mathrm{c} 14 / \mathrm{c} 12=2$. Die Bedeutung weiterer Koeffizientenrestriktionen wird erst in den Simulationen, d. h. im Zusammenspiel aller Gleichungen, ersichtlich. Insgesamt werden (ohne die Koeffizienten deterministischer Variablen) von den 27 aufgelisteten Koeffizienten letztendlich nur neun frei geschätzt.

\section{Das Lohn-Preis-Beschäftigungssystem}

Die makroökonomische Bedeutung der Löhne kommt im NiGEM grundsätzlich innerhalb dreier Wirkungskanäle zum Tragen:

1. Die Lohnentwicklung ist eine Einflussgröße für Anpassungen der Arbeitskräftenachfrage. Eine Erhöhung des Nominallohns kann zwar Preissteigerungen nach sich ziehen (Koeffizienten c23 und c28), deren Einfluss auf den Umsatz der Unternehmen an sich positiv wäre. Das gilt aber nur, falls die abgesetzte Menge konstant bliebe. Vielmehr setzen die vom Lohnniveau abhängigen Kosten direkt Anreize dafür, Arbeitskräfte einzustellen oder zu entlassen (Koeffizienten c35; c36). Neben der gesamtwirtschaftlichen Nachfrage ist die Reallohnentwicklung daher im Ausgangsmodell eine direkt wichtige Einflussgröße auf die Beschäftigungsentwicklung.

2. Die Lohnentwicklung wirkt sich auf die internationale preisliche Wettbewerbsfähigkeit aus, da sich die im Inland determinierten Preissteigerungen (Koeffizienten c23 und c28) über die Verbraucherpreise auch in Exportpreissteigerungen übersetzen (der entspre-
Kasten 1

Lohn-Preis-Beschäftigungssystem

Lohngleichung

$\Delta \log \left(W A G E_{t}\right)=c_{11}+c_{12} \log \left(W_{A G E_{t-1}}\right)+c_{13} \log \left(U T C_{t-1}\right)+c_{14}$ $\log \left(Y_{t-1}\right)+c_{15} T E C H L_{t-1}+c_{16} \log \left(L_{t-1}\right)+c_{17} U_{t-1}+c_{18} \log \left(C E D_{t}\right)$

$+u_{1, t}$

Preisgleichung

$\Delta \log \left(U_{T C} C_{t}\right)=C_{21}+C_{22} \log \left(U T C_{t-1}\right)+C_{23} \log \left(W_{A G E_{t-1}}\right)+C_{24}$ $\log \left(Y_{t-1}\right)+C_{25} T E C H L_{t-1}+C_{26} \log \left(L_{t-1}\right)+C_{27} \log \left(C U_{t-1}\right)+C_{28}$ $\triangle \log \left(W A G E_{t}\right)+u_{2, t}$

Beschäftigungsgleichung

$\Delta \log \left(L_{t}\right)=C_{31}+C_{32} \log \left(L_{t-1}\right)+C_{33} \log \left(Y_{t-1}\right)+C_{34} T E C H L_{t-1}+C_{35}$ $\log \left(W A G E_{t-1}\right)+C_{36} \log \left(U T C_{t-1}\right)+C_{37} \Delta \log \left(Y_{t}\right)+C_{38} \Delta T E C H L_{t}+$ $c_{39} \Delta \log \left(L_{t}\right)+c_{310} \operatorname{\Delta log}\left(W A G E_{t}\right)+c_{311} \operatorname{Vlog}\left(U T C_{t}\right)+D+u_{3, t}$

Variablenverzeichnis: $W A G E=$ nominaler Stundenlohn, UTC $=$ im Inland determinierter Teil der Verbraucherpreise (unit total cost), $Y=$ BIP, TECHL = (das Arbeitsvolumen erweiternder) technologischer Fortschritt, $L=$ Arbeitsvolumen, $U$ = Arbeitslosenquote, $C U=$ Kapazitätsauslastung, $C E D=$ Konsumdeflator, $D=$ Deterministik

chende Langfristkoeffizient beträgt in der Exportpreisgleichung gemäß NiGEM Benutzerhandbuch 0,7; der Kurzfristkoeffizient sogar 0,9). Lohnerhöhungen können zu höheren nominalen Lohnstückkosten führen, wenn die nominalen Arbeitnehmerentgelte stärker steigen als das reale Bruttoinlandsprodukt (BIP). Dieses Verhältnis (Koeffizient c23 im Vergleich zu den Koeffizienten c24 und c26) bestimmt den Druck, den die Arbeitskosten auf das inländische Preisniveau ausüben, was wiederum die Terms of Trade mitbestimmt. Ausgeglichen werden kann diese Entwicklung entweder durch Steigerungen der Lohnstückkosten bei den Handelspartnern oder durch eine Abwertung des nominalen Wechselkurses (außerhalb der Europäischen Währungsunion). Weitere nicht-preisliche Wettbewerbskomponenten sind auch bedeutend (Storm und Naastepad, 2015), werden aber in dem Drei-Gleichungssystem nicht berücksichtigt.

3. Die Löhne sind für die Mehrzahl der Haushalte der wichtigste Bestandteil des verfügbaren Einkommens, und zudem orientieren sich Renten- und Arbeitslosengeldzahlungen vor allem an der Lohnentwicklung. Die Löhne wirken also maßgeblich auf die gesamtwirtschaftliche Nachfrage - sei es durch den privaten Konsum oder durch die privaten Wohnungsbauinvestitionen. 
Nach Pagan (2003) verfolgen Hybrid-Modelle wie NiGEM das doppelte Ziel der theoretischen Nachvollziehbarkeit der Wirkungskanäle sowie der empirischen Konsistenz.

\section{Lohnsimulationen von Sachverständigenrat und Deutscher Bundesbank mit NiGEM}

Die hier diskutierten Simulationsstudien basieren auf kontrafaktischen Simulationen mit dem NiGEM-Modell. Das Modell reproduziert zum einen mehr oder weniger akkurat die tatsächlichen historischen Verläufe aller VGR-VariabIen (Baseline-Simulation). Zum anderen werden bestimmte Variablen (hier der durchschnittliche nominale Lohn pro Stunde) exogen verändert. Die daraus resultierenden Reaktionen der anderen Variablen (Szenario-Simulation) werden dann mit der Baseline verglichen. Diese Abweichungen werden so interpretiert, dass sie die Wirkung von wirtschaftspolitischen Maßnahmen (hier der Lohnerhöhung) zeigen.

Nach dem Ende der Finanzkrise von 2008/2009 und während der Krise des Euroraums warnten SVR und Bundesbank vor stärkeren Lohnsteigerungen in Deutschland. Mit diesen hätten nach Einschätzung einiger ökonomischer Beobachter die Binnennachfrage und damit auch die Importe aus dem europäischen Ausland angekurbelt werden sollen (Niechoj et al., 2010). Damit hätte ein Beitrag zum Abbau der hohen deutschen preislichen Wettbewerbsfähigkeit innerhalb des Euroraums und globalen Leistungsbilanzungleichgewichte geleistet werden können. Anhand von Lohnsimulationen mit NiGEM argumentierten jedoch der SVR (2010) und die Deutsche Bundesbank (2013), dass bei einer solchen Lohnpolitik für Deutschland aufgrund deutlicher Beschäftigungsverluste mittel- und langfristig mit Wachstumsverlusten zu rechnen gewesen wäre, und dass zudem kein positiver Wachstumseffekt für die südeuropäischen Krisenländer aufgetreten wäre.

Die erwähnten Lohnsimulationen von Bundesbank und SVR sind sehr ähnlich, weisen allerdings zwei wesentliche Unterschiede auf: So fällt die exogen gesetzte Erhöhung der nominalen Stundenlöhne bei der Bundesbank mit dauerhaft $2 \%$ über der Baseline doppelt so hoch aus wie beim SVR, und die Bundesbank analysiert einen Zeitraum von 16 Jahren, während es beim SVR nur fünf Jahre sind. Somit fallen die Ergebnisse der Bundesbank bei linearer Spezifikation annähernd doppelt so hoch aus wie die des SVR.

Vergleicht man die gesamtwirtschaftlichen Ergebnisse der beiden Veröffentlichungen für die ersten fünf Jahre, dann zeigen sich in beiden Simulationen - bezogen auf die jeweilige Impulsstärke - ein vergleichbarer Rückgang des realen BIP, ein Anstieg der Verbraucherpreise und ein sofortiger starker Einbruch der Beschäftigung im ersten
Jahr. In beiden Lohnsimulationen kommt es anschlieBend zu einer teilweisen Korrektur des starken Beschäftigungseinbruchs. In der langfristigen Lohnsimulation der Bundesbank findet aber selbst nach 16 Jahren noch keine vollständige Anpassung bei Beschäftigung, Konsum und BIP statt. Diese drei wichtigen Größen verschlechtern sich weiter von Jahr zu Jahr gegenüber der Baseline, sodass sich die langfristigen Effekte nicht eindeutig bestimmen lassen. Dagegen sind die Auswirkungen auf das Wachstum in den Peripherieländern Spanien, Griechenland, Portugal und Irland in beiden Studien vernachlässigbar gering. In den Simulationen von Bundesbank und SVR wird zudem eine sofortige und dauerhafte Erhöhung (keine Senkung) des deutschen Leistungsbilanzüberschusses ausgewiesen.

Die Simulationen des SVR können mit aktuellen NiGEMVersionen gut reproduziert werden (vgl. Abbildung 1, linke Seite). Die Frage der theoretischen Nachvollziehbarkeit wird hier anhand dieser reproduzierten Ergebnisse analysiert. Wie man sieht, übersetzt sich der nominale Lohnschock von $1 \%$ über fünf Jahre in einen Reallohnschock von unmittelbar 0,7\%, der sich nach drei Jahren auf 0,3\% einpendelt. Die Beschäftigung reagiert unmittelbar bei einer restringierten Kurzfristelastizität von über Eins mit einem Abbau um 0,8\%, der sich dann spiegelbildlich zum Reallohn und mit einer Elastizität von de facto Eins auf etwa $-0,3 \%$ einpendelt. Diese schnelle Reaktion ist ökonomisch überraschend, denn üblicherweise würde sich ein solcher Stellenabbau, falls er als Folge eines Verlustes an Wettbewerbsfähigkeit auftritt, erst allmählich vollziehen. Merkwürdig ist zudem, dass die Unternehmen im ersten Jahr diesen Beschäftigungsabbau trotz fast unverändertem Absatz vornehmen können. Denn im ersten Jahr der Simulation bleiben das reale BIP und der Kapitalstock noch nahezu unverändert (Teilabbildung links oben).

Der massive Beschäftigungsabbau um 0,8\% zu Beginn des Simulationszeitraums geht somit automatisch einher mit einem deutlich positiven Produktivitätsschub (Teilabbildung links unten). Da sich gleichzeitig im ersten Jahr die Verbraucherpreise um 0,2\% erhöhen, steigen die Gewinne der Unternehmen spürbar. In den beiden Folgejahren wird diese Entwicklung zur Hälfte korrigiert, weil der Preisauftrieb den Reallohnimpuls gegenüber der Baseline auf 0,3\% reduziert. Dieses Ergebnis bestätigt nicht die Aussage des SVR, dass die „Produktivität in der Simulation jedoch nicht gegenüber dem Basisszenario verändert (wird)“ (SVR, 2010, Ziffer 206). Vielmehr ist das Gegenteil der Fall (Teilabbildung unten links). Im Modell werden institutionelle Faktoren, wie der Kündigungsschutz, der eine derart schnelle und starke Beschäftigungsreaktion der Unternehmen verhindert, offenkundig kaum berücksichtigt. 


\section{Abbildung 1}

Erhöhung der nominalen Stundenlöhne um 1\% ohne geldpolitische Reaktion (für Deutschland)

Veränderung makroökonomischer Größen gegenüber der Baseline in \%
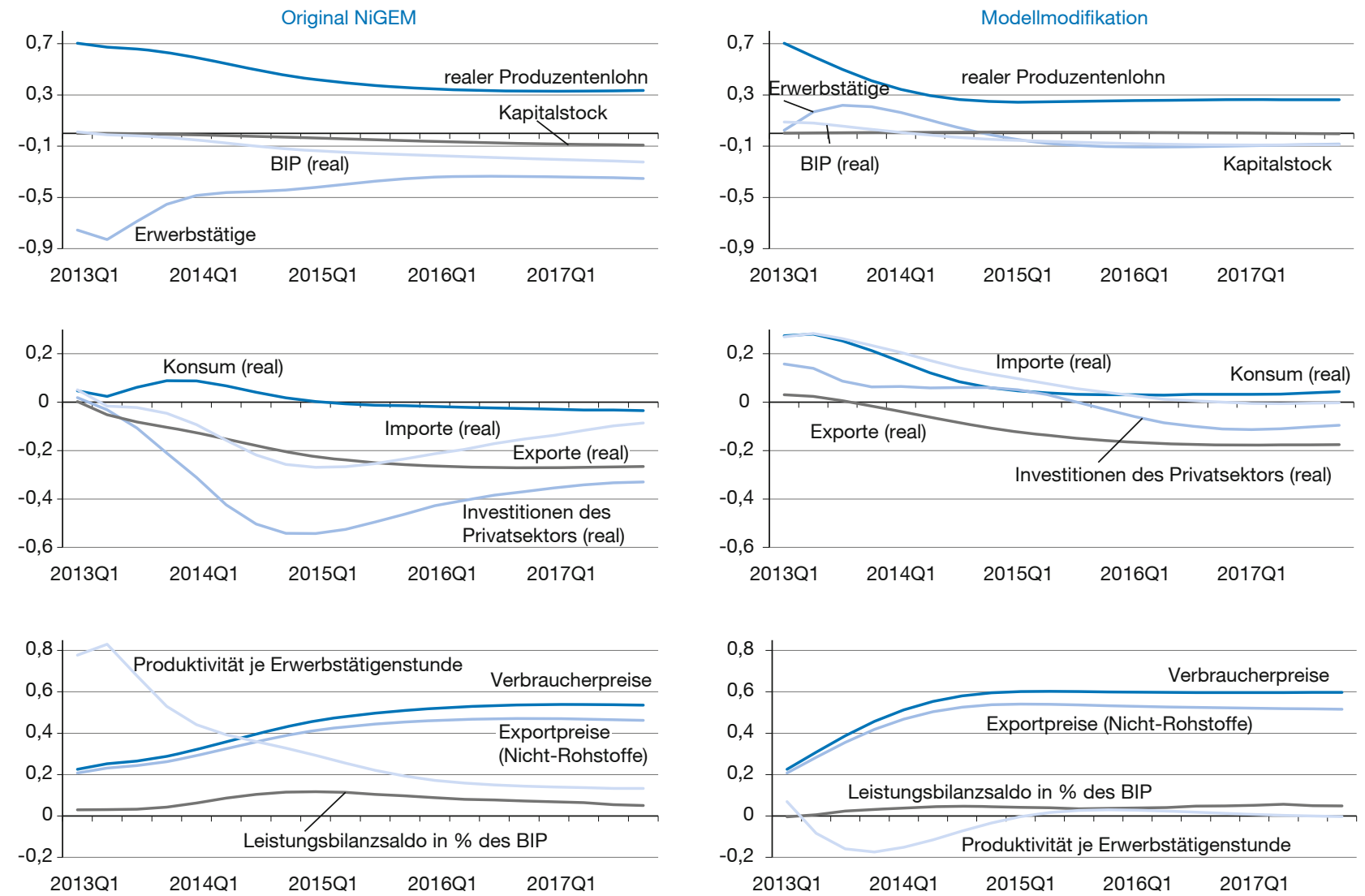

Quelle: eigene Berechnungen.

Aufgrund des sofortigen und starken Beschäftigungseinbruchs in NiGEM nach einer Lohnerhöhung kann der private Konsum kurzfristig kaum zulegen. Darüber hinaus gerät das Wirtschaftswachstum in eine Abwärtsspirale. Da die Exporte aufgrund des Verlustes an preislicher Wettbewerbsfähigkeit rückläufig sind und die Konsumentwicklung wegen der induzierten Beschäftigungsverluste schwach bleibt, wirkt sich dies nachfrageseitig auch negativ auf die reale Investitionsdynamik aus (Teilabbildung links Mitte).

Wie ökonomisch plausibel sind diese Effekte? Wenn es einen Beschäftigungsabbau der Unternehmen nach Lohnkostensteigerungen bei zunächst unverändertem Produktionsniveau gibt, dann bedarf es dazu im Normalfall zusätzlicher (Rationalisierungs-)Investitionen. Die treten im Modell aber nicht auf. Abbildung 1 (links Mitte) zeigt, dass die gesamten privaten Investitionen in NiGEM mit einer Elastizität von gut $-0,5$ im zweiten Jahr des Lohnschocks reagieren. Dies ist der Tatsache geschuldet, dass der schwächeren Exportdynamik bei gegebenem Beschäfti-

gungsabbau keine stärkere binnenwirtschaftliche Dynamik gegenübersteht. Wäre kein Beschäftigungsabbau in solcher Höhe gegeben, dann würden die verfügbaren Einkommen der Haushalte durch den Lohnschock per Saldo nicht belastet, sondern angeregt. Ein großer Teil der Investitionen des Privatsektors in Form von Wohnungsbauinvestitionen dürften dann auch positiv beeinflusst werden.

Das NiGEM-Ausgangsmodell wird also sehr stark durch die jeweils aktuelle Reallohnsituation gesteuert. Induzierte Produktivitätseffekte dürften in der Realität allerdings zu einem großen Teil auf Investitionen und weniger auf Entlassungen beruhen. Dies bleibt in den beschriebenen Simulationen unberücksichtigt. Zudem dürften in der Realität die Beschäftigung bzw. das Arbeitsvolumen viel stärker auf Nachfrageschwankungen reagieren als auf Nominallohnänderungen, zumal letztere in NiGEM in hohem $\mathrm{Maße}$ in den Preisen weitergewälzt werden.

Der SVR weist auch darauf hin, dass „es unzulässig (wäre), die Simulationsergebnisse dahingehend zu interpretieren, 
dass steigende Reallöhne notwendigerweise zu einem Rückgang der Beschäftigung führen“ (SVR, 2010, Ziffer 210). Die Simulationsergebnisse zeigen allerdings das Gegenteil. Auch in Phasen, in denen die Reallöhne letztlich geringer als die Produktivität steigen, kommt es im Modell zu einem Rückgang der Beschäftigung aufgrund der verwendeten Restriktionen. Insgesamt überzeugen die Dynamiken der Simulationen nicht und stellen die Erfüllung des ersten Ziels der theoretischen Nachvollziehbarkeit in Frage.

\section{Die Beschäftigungsgleichung unter ökonometrischer Betrachtung}

Im Folgenden wird nun das zweite Ziel von Hybrid-Modellen wie NiGEM - die empirische Konsistenz - näher betrachtet. In den ersten drei Spalten der Tabelle 1 werden die Variablen der Beschäftigungsgleichung bzw. des Arbeitsvolumens im Arbeitsmarktsystem aufgelistet, die Koeffizientenrestriktionen erläutert und die geschätzten Koeffizienten, wie sie in NiGEM berichtet werden, wiedergegeben. In der Spalte (3) wird die NiGEMSpezifikation des Arbeitsvolumens unter Berücksichtigung der im NiGEM-Benutzerhandbuch aufgeführten Restriktionen im Lohn-Preis-Beschäftigungssystem nachgeschätzt; in der Spalte (4) unter gleichen Bedingungen als Einzelgleichung.

Die Langfristrestriktionen in der NiGEM-Spezifikation sorgen für eine Elastizität von 0,5 zwischen Reallohn und Arbeitsproduktivität. Auf Basis eines Wald-Koeffiziententests und einer Irrtumswahrscheinlichkeit von $5 \%$ kann tatsächlich bei der Systemschätzung nicht ausgeschlossen werden, dass die gewählte Elastizität des Arbeitsvolumens bezüglich des Outputs bei 1, die SemiElastizität bezüglich des technologischen Fortschritts bei $-0,5$ und die Elastizität bezüglich des Reallohns bei $-0,5$ liegen. Eine weitere Langfristrestriktion besteht für den in der Preisgleichung verwendeten Koeffizienten des kapazitätsauslastungsabhängigen Markups, der in Höhe des 1,25-fachen des Ladungskoeffizienten gesetzt wird. ${ }^{1}$ Der Ladungskoeffizient der Langfristbeziehung in der Preisgleichung (c22) wird in NiGEM in gleicher Höhe auch für die Lohngleichung (c12) verwendet. Diese Restriktion lässt sich per Koeffizienten-Test allerdings ablehnen, wenn das Lohn-Preis-Beschäftigungssystem unrestringiert geschätzt wird.

Im Ausgangsmodell wird die Konstante in der Beschäftigungsgleichung (c31) unerklärlicherweise auf einen hohen positiven Wert von 4,96 gesetzt. Das erzeugt einen starken negativen Ladungskoeffizienten. Diese Setzung

1 Diese Restriktion lässt sich zwar nicht auf Basis einer Irrtumswahrscheinlichkeit von $5 \%$, wohl aber von $10 \%$ ablehnen. lässt sich empirisch nicht nachvollziehen. Durch den hohen Ladungskoeffizienten bekommt der Reallohn auch in der kurzen Frist einen stark negativen Einfluss auf die Beschäftigung, da der entsprechende Koeffizient auf das Verhältnis aus den Ladungskoeffizienten der Beschäftigungs- und der Lohngleichung restringiert ist.

Als einziger Kurzfristkoeffizient weist die Lohnveränderung in der Beschäftigungsgleichung eine solche Restriktion auf, die von den Koeffizienten anderer Gleichungen abhängt. Konkret hat diese einen hohen negativen Wert von -1,23. Dieser sehr elastische Zusammenhang kann von der nachgeschätzten Regression nur angenähert werden, wenn man das restringierte System bis 2006 schätzt $(-1,37)$. Die Tatsache, dass es sich unter Berücksichtigung der Restriktionsstruktur nicht um ein stabiles Resultat handelt, wird in Tabelle 1 offenkundig. In der Systemschätzung bis 2018 fällt der Wert sogar auf -1,61 (Spalte 3), da der Ladungskoeffizient der Preisgleichung unter Berücksichtigung der Daten nach 2006 zurückgeht; in der restringierten Einzelgleichungsschätzung (Spalte 4) liegt er dagegen nur bei $-0,37$. Behrend et al. (2019) schätzen in einer kurzfristigen Spezifikation ohne Restriktionen mit einer Langfristbeziehung, bei der die Restriktionen der neoklassischen langfristigen Arbeitsnachfragekurve leicht verändert werden, sogar einen positiven Kurzfristkoeffizienten des Reallohns. Zusammenfassend zeigt die Bandbreite der Resultate, dass bei Verzicht auf die empirisch kaum zu motivierende Restriktion der Kurzfristeinfluss der Löhne auf die Beschäftigung eher klein und teilweise sogar positiv ist.

Zudem fallen die diagnostischen statistischen Tests der System- und der Einzelgleichungsreplikation nicht überzeugend aus. Aufgrund der vielen Restriktionen fällt die Anpassungsgüte gemessen am adjustierten $\mathrm{R}^{2}$ sogar negativ aus. Der t-Wert in Höhe von -3743 bzw. -4730 für den Ladungskoeffizienten c32 deutet auf die Schätzung einer Identitätsbeziehung hin. Dies erlaubt ein erstes Zwischenfazit, nach dem die NiGEM-Ausgangsgleichung keine ökonometrisch bestätigten Zusammenhänge in den simulierten Größenordnungen widerspiegelt.

In Spalte (5) weist die Tabelle 1 die Ergebnisse einer Schätzung ohne jegliche Restriktionen aus. Diese Spezifikation erlaubt eine Einschätzung, inwieweit die Verwendung der neoklassischen Arbeitsnachfragekurve als Langfristbeziehung in der Beschäftigungsgleichung Sinn ergibt. Dabei werden für die Kurzfrist-Koeffizienten Verzögerungen höherer Ordnung zugelassen. Dieses Modell entsteht als Resultat einer sequentiellen Eliminierung der Koeffizienten mit dem höchsten p-Wert, bis nur noch Kurzfrist-Koeffizienten mit p-Wert kleiner als $10 \%$ übrigbleiben. Ersichtlich ist, dass bei der unrestringierten Schätzung die neoklassisch-motivierte Arbeitsnachfrage 
Tabelle 1

Koeffizienten in verschiedenen NiGEM-Modellversionen (1975Q1 bis 2018Q4; 236 Beobachtungen)

\begin{tabular}{|c|c|c|c|c|c|c|c|c|c|c|}
\hline $\begin{array}{l}\text { Abhängige Variable: } \\
\text { DLOG(Arbeitsvolumen) }\end{array}$ & \multirow[t]{3}{*}{$\begin{array}{c}(1) \\
\text { Restriktion }\end{array}$} & \multirow[t]{3}{*}{$\begin{array}{c}\text { (2) } \\
\text { NiGEM }\end{array}$} & $\begin{array}{r}\text { (3) } \\
\text { Replizier } \\
\text { Syster }\end{array}$ & im & \multicolumn{2}{|c|}{$\begin{array}{c}\text { (4) } \\
\text { Repliziert als } \\
\text { Einzelgleichung }\end{array}$} & \multicolumn{2}{|c|}{$\begin{array}{c}(5) \\
\text { Unrestringiert }\end{array}$} & \multicolumn{2}{|c|}{$\begin{array}{c}(6) \\
\text { Modifiziert }\end{array}$} \\
\hline Stichprobe & & & \multicolumn{2}{|c|}{$1975 \mathrm{Q} 1$ bis $2018 \mathrm{Q} 4$} & \multicolumn{2}{|c|}{$1975 \mathrm{Q} 1$ bis $2018 \mathrm{Q} 4$} & \multicolumn{2}{|c|}{ 1975Q1 bis 2018Q4 } & \multicolumn{2}{|c|}{ 1975Q1 bis 2018Q4 } \\
\hline Variablen & & & Koeffizient & t-Wert & Koeffizient & $t-$ Wert & Koeffizient & t-Wert & Koeffizient & t-Wert \\
\hline Konstante & c31 & 4,955 & 4 & -- & 4,955 & -- & 0,346 & 1,03 & 1,635 & 2,70 \\
\hline \multicolumn{11}{|l|}{ Kointegration } \\
\hline LOG(Arbeitsvolumen(-1)) & c32 & $-0,411$ & $-0,412$ & $-3743,25$ & $-0,412$ & $-4730,53$ & 0,001 & 0,04 & $-0,083$ & $-2,74$ \\
\hline LOG(Reales BIP(-1)) & $(-1)^{*} \mathrm{c} 32$ & 0,411 & 0,412 & -- & 0,412 & -- & $-0,056$ & $-1,61$ & 0,083 & -- \\
\hline $\begin{array}{l}\text { Technologischer Fortschritt (- } 1 \text {, } \\
\text { arbeitserw.) }\end{array}$ & $(1-\sigma)^{*} \mathrm{c} 32$ & $-0,206$ & $-0,205$ & -- & $-0,206$ & -- & 0,023 & 1,33 & -- & -- \\
\hline LOG(Realer Produzentenlohn(-1)) & $\sigma^{*} \mathrm{c} 32$ & $-0,206$ & $-0,205$ & -- & $-0,206$ & -- & -- & -- & -- & -- \\
\hline LOG(Nominaler Lohn(-1)) & & -- & -- & -- & -- & -- & 0,031 & 1,06 & -- & -- \\
\hline LOG(im Inland determinierter Preis(-1)) & & -- & -- & -- & -- & -- & $-0,028$ & $-0,80$ & -- & -- \\
\hline LOG(Kapitalstock(-1)) & & -- & -- & -- & -- & -- & -- & -- & $-0,088$ & $-2,66$ \\
\hline \multicolumn{11}{|l|}{ Kurzfrist } \\
\hline DLOG(Reales BIP) & c37 & 0,661 & 0,415 & 4,54 & 0,462 & 5,29 & -- & -- & 0,209 & 4,64 \\
\hline DLOG(Reales BIP(-1)) & & -- & -- & -- & -- & -- & 0,213 & 4,84 & -- & -- \\
\hline DLOG(Reales BIP(-2)) & & -- & -- & -- & -- & -- & 0,182 & 4,33 & -- & -- \\
\hline DLOG(Reales BIP(-3)) & & -- & -- & -- & -- & -- & 0,125 & 3,01 & -- & -- \\
\hline DLOG(Reales BIP(-4)) & & -- & -- & -- & -- & -- & 0,120 & 2,97 & -- & -- \\
\hline$\underline{\mathrm{D} \text { (Technolog. Fortschritt) }}$ & $(-1)^{\star} \mathrm{c} 37$ & $-0,661$ & $-0,415$ & -- & $-0,462$ & -- & -- & -- & -- & -- \\
\hline $\mathrm{D}($ Technolog. Fortschritt(-1)) & & & -- & -- & -- & -- & $-0,155$ & $-1,98$ & -- & -- \\
\hline DLOG(Arbeitsvolumen) & $1-c 37$ & 0,339 & 0,585 & -- & 0,538 & -- & -- & -- & -- & -- \\
\hline DLOG(Beschäftigte(-1)) & & -- & -- & -- & -- & -- & -- & -- & 0,621 & 5,46 \\
\hline DLOG(Realer Produzentenlohn) & $-\sigma^{*} \mathrm{C} 32 / \mathrm{C} 12$ & $-1,231$ & $-1,609$ & -- & $-0,373$ & -- & -- & -- & -- & -- \\
\hline DLOG(Realer Produzentenlohn(-1)) & & -- & -- & -- & -- & -- & -- & -- & 0,114 & 2,04 \\
\hline DLOG(Kapitalstock(-1)) & & -- & -- & -- & -- & -- & -- & -- & $-0,779$ & $-2,15$ \\
\hline Ladungskoeffizient der Lohngleichung & c12 & $-0,167$ & $-0,128$ & $-18,17$ & $-0,552$ & $-3,08$ & -- & -- & -- & -- \\
\hline$(1-\mathrm{S} 91 \mathrm{Q1})^{\star} 0,03 /\left(1+0,035^{\star}(@\right.$ trend-124) & & 1 & $-0,005$ & $-3,13$ & -- & -- & -- & -- & -- & -- \\
\hline S91Q1 & & -- & -- & -- & -- & -- & 0,001 & 0,39 & -- & -- \\
\hline I05Q1 & & -- & -- & -- & 0,025 & 2,73 & -- & -- & 0,026 & 4,74 \\
\hline I97Q2 & & -- & -- & -- & -- & -- & -- & -- & 0,025 & 6,29 \\
\hline adjustiertes $\mathrm{R}^{2}$ & & & \multicolumn{2}{|c|}{$<0$} & \multicolumn{2}{|c|}{$<0$} & \multicolumn{2}{|c|}{0,284} & \multicolumn{2}{|c|}{0,464} \\
\hline Akaike-Informationskriterium & & & \multicolumn{2}{|c|}{--} & \multicolumn{2}{|c|}{$-5,98$} & \multicolumn{2}{|c|}{$-7,90$} & \multicolumn{2}{|c|}{$-7,53$} \\
\hline Schwarz-Bayes-Informationskriterium & & & \multicolumn{2}{|c|}{--} & $-5,91$ & & $-7,6$ & & $-7,3$ & \\
\hline Hannan-Quinn-Informationskriterium & & & -- & - & $-5,95$ & & $-7,8$ & & $-7,2$ & \\
\hline Diagnose-Test: Residuen & Null-Hypo & these & $\mathrm{p}$-Wer & & $\mathrm{p}$-Wer & & $p-W$ & & $\mathrm{p}-\mathrm{W}$ & \\
\hline Breush-Godfrey LM(1) & \multirow{8}{*}{\multicolumn{2}{|c|}{$\begin{array}{l}\text { Keine Residuen- } \\
\text { Autokorrelation }\end{array}$}} & 0 & & $0,97 \varepsilon$ & & 0,6 & 18 & 0,0 & \\
\hline Breush-Godfrey LM(2) & & & 0 & & & 0 & 0,6 & 86 & 0,9 & \\
\hline Breush-Godfrey LM(3) & & & 0 & & & 0 & 0,5 & 63 & 0,0 & \\
\hline Breush-Godfrey LM(4) & & & 0 & & 0,002 & & 0,6 & 99 & 0,6 & \\
\hline Breush-Godfrey LM(5) & & & 0 & & 0,015 & & 0,8 & 08 & 0,0 & \\
\hline Breush-Godfrey LM(6) & & & 0 & & 0,024 & & & 45 & 0,0 & \\
\hline Breush-Godfrey LM(7) & & & 0 & & 0,014 & & & 63 & 0,0 & \\
\hline Breush-Godfrey LM(8) & & & 0 & & 0,144 & & & 49 & 0,3 & \\
\hline White w/o cross terms & Homoskeda & astizität & -- & & 0,007 & & & 0 & 0,0 & \\
\hline Jarque-Bera & Normalvert & teilung & 0 & & 0,02 & & 0,0 & 01 & 0,0 & \\
\hline Ramsey Reset (2 terms) & Linearit & & -- & & -- & - & 0,2 & 77 & 0,5 & \\
\hline
\end{tabular}

Mit $\sigma=0,5$ wird in NiGEM die Substitutionselastizität zwischen Arbeit und Kapital bezeichnet.

Quelle: eigene Berechnungen. 
in der Kointegrationsbeziehung der Gleichung jeglichen Erklärungsgehalt verliert. Der Ladungskoeffizient ist vergleichsweise klein, positiv und insignifikant. Das deutet darauf hin, dass die oben genannten Testergebnisse zu den Elastizitäten und Semielastizitäten der Langfristbeziehungen von den Kurzfristsetzungen abhängen. Damit wird das Zwischenfazit nochmals bekräftigt.

Als Konsequenz wird im Folgenden gezeigt, wie eine Modifizierung der NiGEM-Beschäftigungsgleichung (Tabelle 1, Spalte 6), die das Ziel der empirischen Konsistenz besser erfüllt, die Reaktion der Beschäftigungsvariable auf Lohnänderungen verändert und zudem die theoretische Nachvollziehbarkeit verbessert.

\section{Effekte einer Modifikation der \\ Beschäftigungsgleichung}

Tabelle 1 zeigt in der Spalte (6) eine sowohl keynesianisch als auch empirisch motivierte Modifikation der Langfristbeziehung und der Kurzfristmodellierung. Diese modifizierte Gleichung ist angelehnt an die Beschäftigungsgleichung des makroökonometrischen Modells des IMK (Herr et al., 2018). In der langen Frist bilden nur das BIP und der Kapitalstock eine Kointegration mit der Beschäftigung. Kointegrationstests zeigen, dass sich die Kointegrationsbeziehung in der Beschäftigungsgleichung auf eine Spezifikation ohne die Reallöhne reduzieren lässt. Als einzige Restriktion wird eine Nachfrageelastizität des Arbeitsvolumens in Höhe von 1 aufrechterhalten, die sich im Koeffizienten-Test bei einer Irrtumswahrscheinlichkeit von $5 \%$ nicht ablehnen lässt. Qualitativ fallen die Schätzund Simulationsergebnisse zudem ähnlich aus, wenn man auch diese Restriktion aufgibt. Im Gegensatz zur NiGEM-Ausgangsgleichung und der Schätzgleichung, in der die Restriktionen aufgelöst werden, sind nun alle Koeffizienten signifikant und somit empirisch motiviert. Auch die diagnostischen Tests zeigen ein besseres Ergebnis, auch wenn noch Schwachstellen in Form von serieller Korrelation bestehen. Zusammenfassend ist die Langfristbeziehung empirisch durch Signifikanz- und Kointegrationstests validiert; wirtschaftstheoretisch lässt sich die Spezifikation als keynesianisch einordnen.

Denn eine keynesianisch motivierte Beschäftigungsgleichung erfordert, dass die Entscheidung über die Arbeitsnachfrage dem Gütermarkt nachgelagert getroffen wird. Grundsätzlich hängt daher die Arbeitsnachfrage stark vom Wirtschaftswachstum ab. Produktivitätseffekte, die diesen Zusammenhang verändern, entstehen unter anderem durch zusätzliche Investitionen in den Kapitalstock. Die Lohnentwicklung wirkt auf die Arbeitsnachfrage ein, indem sie Einfluss auf das Wirtschaftswachstum, die Preise und auf die Investitionstätigkeit hat. Die Unterneh- men können kurzfristig nicht „beliebig“ zwischen Arbeit und Kapital substituieren, sondern nur in dem Maße, wie sie vorher ihre Investitionen arbeitssparend ausgerichtet haben. Investitionen wirken dann als Komponente des BIP vor allem kurzfristig nachfrage- und beschäftigungssteigernd, über die Erhöhung des Kapitalstocks produktivitätssteigernd und daher für sich genommen potenziell vor allem langfristig beschäftigungsmindernd.

Abbildung 1 (rechte Seite) illustriert Ergebnisse des ursprünglichen Simulationsdesigns des SVR (2010) mit der modifizierten Beschäftigungsgleichung in NiGEM. ${ }^{2}$ Die Teilabbildung oben rechts zeigt, dass sich der Reallohnschock in dieser Modellvariante ähnlich entwickelt wie in der reproduzierten Simulation: Die Reallöhne steigen um ca. $0,7 \%$ gegenüber der Baseline, um sich nun schon nach zwei Jahren auf zusätzliche 0,3\% einzupendeln. Die Beschäftigung reagiert aber ganz anders: Bis zur Mitte des zweiten Jahres wird ein leichter und allmählicher Ausbau gegenüber der Baseline simuliert; danach dreht sich die Wirkung vernachlässigbar gering ins Negative.

Auch Kapitalstock und BIP verändern sich im Gegensatz zur Simulation des SVR kaum. Was sich verändert, ist die Zusammensetzung des Wachstums (Teilabbildung Mitte). Während die realen Exporte wie in der Simulation des SVR rückläufig gegenüber der Baseline sind, liefert der reale private Verbrauch nunmehr eine positive Veränderung. Das stützt nachfrageseitig auch die realen Investitionen, die sich in der ersten Hälfte des Simulationszeitraums schwach positiv gegenüber der Baseline und in der zweiten Hälfte schwach negativ entwickeln. Anders als in der Simulation des SVR nehmen die Importvolumina gegenüber der Baseline zu. Die Teilabbildung unten rechts zeigt, dass sich zudem geringe Veränderungen der Produktivität und des Leistungsbilanzsaldos ergeben. Dass der Leistungsbilanzsaldo nicht rückläufig gegenüber der Baseline ist, dürfte auf das Anziehen der Exportpreise zurückzuführen sein.

Zusammenfassend: Mit modifizierter Beschäftigungsgleichung liefern höhere Lohnpfade im NiGEM-Modell entgegen den Simulationen von Sachverständigenrat und Deutscher Bundesbank keine signifikanten Wachstums- und Beschäftigungsverluste gegenüber der Baseline. Vielmehr regt die ausgewogenere Verteilung zugunsten der Arbeitseinkommen die Binnennachfrage an und bietet aufgrund stärkerer Steuereinnahmen zusätzlichen fiskalischen Spielraum zum Abbau der außenwirtschaftlichen Ungleichgewichte innerhalb des Euroraums (Lindner, Stephan und Zwiener, 2018).

2 Ähnlich dem Vorgehen von Behrend et al. (2019) werden zudem neu geschätzte Import- und Exportgleichungen für Deutschland verwendet. Als wichtiges Resultat zeigt die aktualisierte Schätzung eine verringerte Nachfrageelastizität der Importe. 


\section{Schlussfolgerungen}

Die eingangs diskutierten Modellsimulationen von Sachverständigenrat und Deutscher Bundesbank mit NiGEM zu den vermeintlich negativen gesamtwirtschaftlichen Auswirkungen von Lohnerhöhungen dienten der wirtschaftspolitischen Beratung. Sie wurden publiziert, als in Deutschland der Ruf nach stärkeren Lohnsteigerungen nach einer langen Phase der Lohnmoderation in den 2000er Jahren lauter wurde und sich die Europäische Währungsunion aufgrund interner Wettbewerbsungleichgewichte in einer schwierigen Phase befand. Insofern kam den Empfehlungen von Bundesbank und SVR in dieser Zeit Bedeutung zu.

Die Simulationen wurden mit einem Modell durchgeführt, das in seinem Kern, dem System für die Lohn-, Beschäftigungs- und Preisgleichung, nicht allein auf empirisch geschätzten Gleichungen, sondern stark auf gesetzten Restriktionen beruhte und auch heute noch beruht. Bei der Überprüfung der empirischen Relevanz dieser Setzungen zeigt sich, dass selbst das 2006 implementierte zentrale Gleichungssystem zum damaligen Zeitpunkt statistisch nicht wirklich abgesichert werden konnte. Bestimmte Setzungen, wie z. B. die Konstante in der Beschäftigungsgleichung oder die Restriktion für den kurzfristigen Einfluss des Reallohns, hatten einen starken Einfluss auf die Koeffizientenschätzungen der erklärenden Variablen. In diesem Beitrag wird anhand von Simulationen gezeigt, dass sowohl das Ziel der Nachvollziehbarkeit der Wirkungskanäle, insbesondere in der kurzen Frist, als auch die empirische Konsistenz nicht ausreichend gegeben sind. Dies ruft nach einer Modifikation des Modells. Zudem dürfte vielen Nutzern von NiGEM die konkrete Ausgestaltung des Systems, die Implikationen der Setzungen, aber auch deren statistische (In)Signifikanz nicht bekannt (gewesen) sein.

In diesem Artikel wird eine Modifikation des Modells ohne Kurzfristrestriktionen und mit einer alternativen - keynesianisch inspirierten - Kointegrationsbeziehung durchgeführt, deren empirische Validität besser abschneidet als die des NiGEM-Ausgangsmodells. Mit dieser Modifikation verändern sich auch die Ergebnisse und die wirtschaftspolitischen Aussagen der erwähnten Simulationen des SVR und der Deutschen Bundesbank: Ein höherer Lohnpfad regt nun stärker die Binnennachfrage an, weil die "gesetzten" unmittelbaren Beschäftigungsverluste ausbleiben. Merkliche Wachstums- und Beschäftigungsverluste für die deutsche Volkswirtschaft bleiben im gesamten Simulationszeitraum aus - vielmehr ändert sich die Zusammensetzung der Wachstumskräfte zugunsten der Binnennachfrage. Zusätzlicher fiskalischer Spielraum aufgrund stärkerer Steuereinnahmen im Inland bietet die Möglichkeit zu einer Wachstumspolitik, die zum Abbau der außenwirtschaftlichen Ungleichgewichte innerhalb des Euroraums beitragen kann.

Mit den Restriktionen des NiGEM-Ausgangsmodells und inrer Beibehaltung über eineinhalb Jahrzehnte wurde ein vielfach in der wirtschaftspolitischen Beratung zitiertes Ergebnis konzipiert, das empirisch kaum gerechtfertigt werden kann.

\section{Literatur}

Behrend, A., K. Gehr, C. Paetz, T. Theobald und S. Watzka (2019), Europa kann es besser: Wirtschaftspolitische Szenarien für stabileres Wachstum und mehr Wohlstand, Friedrich-Ebert-Stiftung.

Deutsche Bundesbank (2013), Zu den makroökonomischen Effekten einer Anhebung des Lohnniveaus in NiGEM Simulationen, Monatsbericht, Februar.

Hantzsche, A., M. Lopresto und G. Young (2018), Using NiGEM in uncertain times: Introduction and overview of NiGEM, National Institute Economic Review, Nr. 244.

Herr, H., A. Herzog-Stein, J. Kromphardt, C. Logeay, P. Nüß, T. Pusch, T. Schulten, A. Watt und R. Zwiener (2018), Makroökonomische Folgen des gesetzlichen Mindestlohns aus keynesianisch geprägter Perspektive, Studie im Auftrag der Mindestlohnkommission, Endbericht, veröffentlicht bei der Mindestlohnkommission, 59 f. und Anhang A4.

Lindner, F., S. Stephan und R. Zwiener (2018), Dringend gebraucht - aktive Wirtschaftspolitik, um Außenhandelsüberschüsse abzubauen, Wirtschaftsdienst, 98(9), 644-650.

Niechoj, T., U. Stein, S. Stephan und R. Zwiener (2011), Deutsche Arbeitskosten: Eine Quelle der Instabilität im Euroraum, Auswertung der Eurostat-Statistik für 2010, IMK Report, Nr. 68.

Pagan (2003), Report on modelling and forecasting at the Bank of England, Bank of England Quarterly Bulletin, Spring, 60-88.

Sachverständigenrat zur Begutachtung der gesamtwirtschaftlichen Entwicklung (SVR) (2010), Auswirkungen einer expansiven Lohn- und Fiskalpolitik in NiGEM, Jahresgutachten 2010/11.

Sims, C. A. (1980), Macroeconomics and Reality, Econometrica, 48(1), 148.

Storm, S. und C. W. M. Naastepad (2015), Crisis and recovery in the German economy: The real lessons, Structural Change and Economic Dynamics, 32, 11-24.

Title: Wage Levels and Employment - On Economic Policy Advice Using the Multi-Country Model NiGEM

Abstract: The restrictions of the NiGEM initial model and their relevance over the last decade and a half have produced oft-cited economic policy advice that can hardly be justified empirically: Wage increases will result in significant employment losses. But with statistically validated modifications, the results and the economic policy statements of the simulations by the German Council of Economic Experts and the Deutsche Bundesbank find that a higher wage path now stimulates domestic demand more strongly because the set employment losses do not materialise. There are no noticeable losses in growth and employment for the German economy.

JEL Classification: C54, E24, E27 